Natural Product Communications

\title{
The Chemical Composition and Antibacterial Activities of the \\ Essential Oils from Three Aframomum Species from Cameroon, and Their Potential as Sources of $(E)-(R)$-Nerolidol
}

\author{
Sylvie Kwanga Nguikwie ${ }^{\mathrm{a}, \mathrm{b}, \mathrm{e}}$, Maximilienne A. Nyegue ${ }^{a}$, Florentine Ndoye-Foe Belinga ${ }^{\mathrm{a}}$, \\ Rosalie A. Ngono Ngane ${ }^{b}$, Bernard Romestand ${ }^{c}$, Achraf Kouzayhac, Hervé Casabianca ${ }^{d}$, \\ Paul H. Amvam Zollo" and Chantal Menut ${ }^{e *}$
}

\author{
${ }^{a}$ Laboratoire de Phytobiochimie et d'Etude des Plantes Médicinales, Faculté des Sciences, BP 812 Yaoundé, \\ Cameroun \\ 'Laboratoire de Biochimie, Faculté des Sciences, BP 24157 Douala, Cameroun
}

'Equipe Réponse Immunitaire des Macroorganismes et Environnement (RIME): UMR 5I19 (UMII, IFREMER, CNRS), CC 080, Université Montpellier 2, Place E. Bataillon, 34095 Montpellier cedex 05, France

${ }^{`}$ Service Central d'Analyse, Institut des Sciences Analytiques, 5 rue de la Doua, 69100, Villeurbanne, France

"Equipe "Glyco et nanovecteurs pour le ciblage thérapeutique", Institut des Biomolécules Max Mousseron, Faculté de Pharmacie, 15 Avenue Charles Flahault, BP 14491,34093 Montpellier France

chantal.menut@univ-montp2.fr

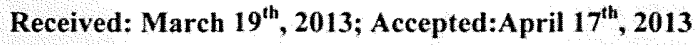

Essential oils obtained by hydrodistillation of seeds, pericarps, leaves and rhizomes of Aframomum dalzieli, A. letestuiamum and A. pruinosum grown in Cameroon were analyzed by GC-FID and GC-MS. The seed oils of the three species were characterized by a high content of $(E)-(R)$-nerolidol $(>88.0 \%)$, which was fully characterized by NMR spectroscopy and chiral GC analysis. The main constituents of the pericarp and rhizome oils were monoterpene hydrocarbons, mainly $\beta$-pinene $(0.8 \%-22.9 \%)$ and sabinene $(29.0 \%-42.3 \%)$, along with 1,8 -cineole $(4.5 \%-23.7 \%)$; leaf oils were characterized by sesquiterpenes, namely $(E)-\beta$-caryophyllene $(18.4 \%-82.4 \%)$ and caryophyllene oxide $(4.5 \%-23.7 \%)$. The antibacterial activities of these essential oils and of nine pure compounds (sabinene, $\beta$-pinene, 1,8-cineole, linalool, racemic $(E)$-nerolidol, $(E)-(R)$-nerolidol, $(E)$ - $\beta$-caryophyllene, $\alpha$-humulene and caryophyllene oxide) were assessed against Micrococcus luteus and Escherichia coli. The strongest activities were observed against $E$. coli. The seed essential oils and their major component, $(E)-(R)$-nerolidol, exhibited the lowest $\mathrm{MIC}$ values $(0.19-0.39 \mu \mathrm{L} / \mathrm{mL})$, justifying their traditional use and their potential application as natural food preservatives.

Keywords: Aframomum dalzielii, Aframomum letestuianum, Aframomum pruinosum, Zingiberaceae, Essential oils, $(E)-(R)-N e r o l i d o l$, Antibacterial activity, Natural preservative.

The therapeutic potentials of aromatic and medicinal plants and of their extracts have been recognized since antiquity; their pharmaceutical properties are partially attributed to their volatile aroma components, composed mainly of terpenes, in addition to other non-terpene components that are currently extracted from the plant material by water or steam distillation. Numerous biological properties of essential oils have recently been reviewed [1-3]. Among these, the antimicrobial qualities of the essential oils from a wide variety of plants, as well as those of their constituents, have been assessed [4,5]. In spite of these data, the greatest use of essential oils is still in food (as flavorings), perfumes and pharmaceutical products for their functional properties. Up to now, only a few preservatives containing essential oils are commercially available [6]. In the meantime, there is a growing number of outbreaks of food-borne illness caused by less than 20 different bacterial species. Due to the resistance that some microorganisms have built up against antibiotics, there is a need for new biologically active components for the maintenance or extension of product shelf life [7]; spices that have been recognized for their antimicrobial properties [8] might represent interesting sources of additives for the reduction or elimination of food-bome pathogens.

The genus Aframomum, family Zingiberaceae, is mainly spread in the rainy forest areas of Africa. Over 20 species of this genus are found in Cameroon, where they are widely used for medicinal, ethno-dietary, cultural and spiritual purposes [9a]. Aframomum species are well known in several countries for their odoriferous leaves and fruits with aromatic seeds which produce essential oils [9a, 10]. Among them, A. melegueta (Rosc.) K. Schum., called "maniguette" or "grains of paradise", is probably the most studied [11a]; its seeds contain the same pungent principles as ginger (6paradol, gingerol and shogaol), which justifies their use as spices and their other designation as "Poivre de Guinée" [12].

As a contribution to our investigations on volatiles from Aframomum species growing in Central Africa [9a-9b, 1la-11b], we have focused on three species as potential sources of $(E)$-nerolidol in their seed essential oils: A. dalzielii Hutchinson (synonym: A. leptoplepis), A. letestuianum Gagnepain and A. pruinosum Gagnepain. In the eastern region of Cameroon, these species grow in the wild, while they are highly cultivated in the western highlands for the local trading of their fruits. These contain sweet and spicy seeds with pleasant scent, popularly consumed in culinary preparations. They are used as ingredients in "medicines" to cure several afflictions like sterility for women and schizophrenia, as reported by traditional healers; they might also have antisympathetic properties and tranquilizing effects [13]; there is a general agreement on their properties to bring strength and peace in families with twins (data not published). 
Regarding chemistry, Tane et al. [13] reported on eleven Aframomum species collected in Cameroon (among them $A$. letestuianum and $A$. pruinosum), which contained diterpenoids, flavonoids, sesquiterpenoids and arylalkanoids. To the best of our knowledge there were no previous chemical investigations on A. dalzielii. Finally, the only chemical investigations on essential oils from Aframomum species collected in Cameroon were performed on nineteen species from various geographical areas [9a]; all the essential oils contained mainly terpenic components obtained from the shikimic acid pathway, usually found in essential oils, but in minor amounts; among them, A. letestuianum and A. pruinosum seed oils were rich in $(E)$-nerolidol $(67 \% ; 91.7 \%)$ respectively, while $\alpha$-pinene $(31.4 \%)$ and $(E)$ - $\beta$-caryophyllene $(25.8 \%)$ were the main constituents of $A$. pruinosum leaf oil.

The cultural and medicinal interest attributed to the Aframomum species growing in Cameroon increases the commercial value of their fruits. To enhance the value of these species, we have systematically investigated the chemical constituents of the essential oils obtained from seeds, pericarps, leaves and rhizomes of A. dalzielii, A. letestuianum and $A$. pruinosum; the antibacterial activities of these essential oils, as well as those of their main components, were tested against two bacterial species: Micrococcus luteus, a Gram-positive human pathogen, and Escherichia coli (Gram-negative), a foodborne bacterium, also implicated in nosocomial infections.

Pericarps, seeds, leaves and rhizomes of the three species were submitted to hydrodistillation; the highest yields were obtained from pericarps $(0.3-2.2 \%)$, followed by seeds $(0.1-0.7 \%)$; the lowest contents of volatile components were observed in the rhizomes $(0.06-0.1 \%)$, while the leaves of the three species afforded almost the same amounts of essential oils $(0.1-0.3 \%)$. Globally, the best yields were obtained from $A$. pruinosum fruits (Table 1).

Table 1: Oil yields from different parts of three Aframomum species collected in Cameroon

\begin{tabular}{|c|c|c|c|c|c|c|}
\hline \multirow[t]{2}{*}{ Species } & \multirow[t]{2}{*}{ Date of harvest } & \multirow[t]{2}{*}{ Locality } & \multirow[b]{2}{*}{ Seeds } & \multicolumn{3}{|c|}{ Oll yields (w/w) } \\
\hline & & & & Pericarps & Leaves & Rhizomes \\
\hline A. dolzielii & $\begin{array}{l}\text { August } 26 \text { th } \\
2008\end{array}$ & Fontem & 0.6 & 1.2 & 0.3 & 0.06 \\
\hline $\begin{array}{c}\text { A. } \\
\text { pruinasturn }\end{array}$ & $\begin{array}{l}\text { August 26th } \\
2008\end{array}$ & Fontem & 0.7 & 2.2 & 0.1 & 0.1 \\
\hline $\begin{array}{c}\text { A } \\
\text { letexztiantem }\end{array}$ & March I 5th 2008 & Bamendjida & 0.1 & 0.3 & 0.2 & 0.1 \\
\hline
\end{tabular}

$\mathrm{GC}$ and GC-MS analyses of the essential oils allowed the identification of 73 components, amounting to $89.7-99.7 \%$ of the total chemical composition (Table 2). Important qualitative and quantitative variability was noticed according to the plant part and/ or the botanical species; nevertheless, the seed oils were characterized by the same major component, $(E)$-nerolidol (88.0$95.1 \%$ ), which could find its application as an agroalimentary product and in perfumery for its long lasting floral odor [14, 15].

This component was, therefore, isolated by liquid solid chromatography from the seed essential oil of $A$. dalzielii and fully characterized by ${ }^{1} \mathrm{H}$ and ${ }^{13} \mathrm{C}$ NMR spectroscopy. The data, which were in agreement with the literature, confirm the formal identification of $(E)$-nerolidol as the major component of the seed essential oil [16]; the specific optical rotation of this component was measured as $[\alpha]_{D}=-13.8\left(c 0.1, \mathrm{CHCl}_{3}\right)$. Analysis by gas chromatography on a chiral column compared with that of the racemic sample (resolution factor 1.01$)$ and $(+)-(E)$-nerolidol from Cabreuva oil (Myrocarpus fastigiatus) used as reference, indicated an enantiomeric excess of the levorotary $(E)$-nerolidol $(96.4 \%)$; chiral analyses of the seed essential oils of $A$. letestuiamum and $A$. pruinosum, performed under the same experimental conditions, indicated an enantiomeric excess of $(-)-(E)$-nerolidol in both samples (respectively $99.5 \%$ and $98.3 \%$ ). These results confirm those previously obtained with the seed oil of $A$. pruinosum collected in Cameroon [1 $1 \mathrm{~b}]$.

The accurate quantification of $(E)$-nerolidol in the seed oils was performed using a multilevel standardization method with tridecane as internal standard (see Experimental). The values obtained $(\mathrm{g} / 100 \mathrm{~g}$ of the seed oil) were $75.6 \pm 0.3$ for $A$. letestuianum; $84.6 \pm$ 0.4 for $A$. dalzielii; and $88.3 \pm 0.4$ for $A$. pruinosum.

The known natural sources of this component are not so numerous: the same enantiomer was identified in the wood oil of Dalbergia parviflora (Leguminosae) [14]; on the other hand, the $(+)-(S)$ enantiomer is predominant in Cabreuva oil; finally, $(E)$-nerolidol is also the main headspace component of Cymbidium goeringii var. goringii, a species with cultural importance in Japan and Southern China, to which it provides a lily of the valley type contribution to the pleasant scent [17].

As previously published by Amvam Zollo et al. [9a], the leaf essential oil of $A$. pruinosum was dominated by sesquiterpenes, mainly $\beta$-caryophyllene, $\alpha$-humulene and caryophyllene oxide $(47.7 \%, 3.0 \%$ and $6.2 \%)$; these components remain the main volatile constituents of $A$. letestuianum $(18.4 \%, 12.4 \%$ and $23.7 \%)$ and $A$. dalzielii leaves $(81.4 \%, 4.3 \%$ and $3.3 \%)$, which are described here for the first time.

As far as the literature survey could ascertain, pericarp and rhizome essential oils from the three species have never been reported before. The essential oils from pericarps were rather dominated by monoterpenes, mainly sabinene and $\beta$-pinene $(31.9 \%-56.5 \%$ all together) in variable relative proportions, along with 1,8 -cineole (10.6\%-14.0\%); a relatively high content of linalool $(10.1 \%)$ characterized the essential oil from $A$. letestuianum pericarps. Finally, the essential oils obtained from thizomes, in all cases in very low yields, are very similar to those of the pericarp extracts; they are dominated by pinenes and sabinene, but with some qualitative and quantitative differences regarding the minor components.

The results of the antibacterial tests performed on all twelve essential oil samples are reported in Table 3 ; the MIC values varied from 0.19 to $50 \mu \mathrm{L} / \mathrm{mL}$.

Globally, the strongest activities were obtained against the Gramnegative bacterium $E$. coli, with MICs varying from 0.19 to 1.56 $\mu \mathrm{L} / \mathrm{mL}$ according to the essential oil sample, while the antimicrobial activities against $M$. luteus were lower (MICs 0.39 to $50 \mu \mathrm{L} / \mathrm{mL}$ ). These activities could be classified in three levels: Strong activity for MIC value ranging from 0.19 to $0.78 \mu \mathrm{L} / \mathrm{mL}$; Moderate activity in the range 1.56-6.25 $\mu \mathrm{L} / \mathrm{mL}$; and Low activity between 12.5 and $50 \mu \mathrm{L} / \mathrm{mL}$.

Considering the above, the essential oils tested showed either strong or moderate activity against $E$ coli, the most efficient samples being seed oils, along with the rhizome oils from $A$. dalzielii and $A$. pruinosum, and the volatile extract from $A$. letestuianum pericarps. The seed oils were the most efficient samples against $M$. luteus, with MICs ranging from 0.39 to $0.78 \mu \mathrm{L} / \mathrm{mL}$; all the other samples showed weak inhibitory activity against $M$. luteus, except for the leaf oil of $A$. letestuianum (MIC $=0.78 \mu \mathrm{L} / \mathrm{mL})$. A strong bactericidal activity against both microorganisms was observed with $A$. letestuianum seed oil (MBC $=0.39-0.78 \mu \mathrm{L} / \mathrm{mL})$. 
Table 2: Comparative percentage composition of essential oils from seeds, pericarps, leaves and rizomes of $A$. dalsielii, A. letestuiamum and $A$. prainosum.

\begin{tabular}{|c|c|c|c|c|c|c|c|c|c|c|c|c|c|c|}
\hline & & \multicolumn{3}{|c|}{ A. dalzielii } & & & etestutian & & & & & mosum & & Identification methods \\
\hline $\begin{array}{l}\text { ldentified comnounds } \\
\text { Monoterpenes }\end{array}$ & LRI & $\begin{array}{l}\mathrm{Se} \\
1.6 \\
\end{array}$ & $\frac{\mathrm{Pe}}{91.1}$ & $\frac{L E}{5.0}$ & $\frac{\mathrm{Rh}}{79.7}$ & $\frac{\mathrm{Sr}}{2.8}$ & $\begin{array}{c}P_{R} \\
82.9 \\
\end{array}$ & $\frac{16}{17.3}$ & $\frac{\mathrm{Bh}}{75.2}$ & $\frac{\mathrm{Se}}{2.0}$ & $\frac{\mathrm{Pe}}{82.9}$ & $\frac{16}{34.7}$ & $\frac{\mathrm{Rh}}{79.4}$ & $\frac{1}{1}$ \\
\hline Monoterpene hydrocarbons & & 1.0 & 70.7 & 4.6 & 71.9 & 0 & 50.7 & 15.9 & 58.0 & 1.6 & 49.5 & 33.5 & 57.6 & $T$ \\
\hline $\begin{array}{l}\text { a-Thuiene } \\
\text { a-Pinene }\end{array}$ & $\begin{array}{l}928 \\
938\end{array}$ & 7 & $\begin{array}{l}0.3 \\
7.8\end{array}$ & $\begin{array}{c}1 \\
0.5\end{array}$ & $\begin{array}{l}0.4 \\
7.8\end{array}$ & $\frac{7}{1}$ & $\begin{array}{l}0.9 \\
6.4\end{array}$ & $\begin{array}{l}1 \\
2.2\end{array}$ & $\begin{array}{l}0.6 \\
5.9\end{array}$ & $\frac{7}{1}$ & $\begin{array}{l}0.7 \\
5.9\end{array}$ & 3.9 & $\begin{array}{l}1.9 \\
5.2\end{array}$ & $\begin{array}{c}\text { MS. LRI } \\
\text { GC, MS. LRI }\end{array}$ \\
\hline Camphene & 952 & i & 0.1 & $i$ & 0.1 & $i$ & 1 & $i$ & 1 & $i$ & 1 & 1.1 & 1 & MS, LRI \\
\hline Sabinene & 977 & 1 & 14.2 & $\mathrm{t}$ & 22.9 & 1 & 0.8 & 1 & 11.6 & 1 & 2.9 & 0.3 & 9.8 & GC. MS. LRI \\
\hline ß-Pinene & 985 & 1 & 42.3 & 3.9 & 37.9 & j & 38.5 & 13.4 & 32.9 & f & 29,0 & 27,8 & 34.3 & GC, MS, LRI \\
\hline Myrcene & 988 & 0.1 & 0.5 & 1 & 0.6 & 1 & $t$ & 1 & 0.5 & 1 & 1 & 1 & 1 & MS, LRI \\
\hline a-Terpinene & 1018 & 1 & 0.6 & ! & 1 & 1 & $t$ & 1 & 1.1 & 1 & 1 & ! & 0.5 & MS, LRI \\
\hline p-Cymene & 1022 & 1 & 0.5 & 1 & 0.3 & 1 & 1.2 & 1 & 0.5 & 1 & 4.0 & 1 & 2.7 & MS. L.RI \\
\hline Phellandrene & 1030 & 0.1 & 1 & 1 & 1 & 1 & & $i$ & 1 & $i$ & & $i$ & 1 & MS. LRI \\
\hline Limonene & 1030 & 1 & 2.7 & 0.2 & 1.6 & 1 & 2.5 & 0.3 & 2.2 & 1 & 7.0 & 0.4 & 1.5 & GC.MS, LRI \\
\hline (Z)-B-Ocimene & 1045 & 0.4 & 1 & 1 & 1 & 1 & 1 & 1 & 0.1 & 1 & 1 & 1 & 1 & MS, LRI \\
\hline (6)-B-Ocimene & 1050 & 0.4 & 1 & 1 & 1 & 1 & 1 & 1 & $i$ & 1.6 & $y$ & 1 & 1 & GC. MS, LRI \\
\hline$\gamma$-Terpinene & 1057 & $i$ & 1.3 & 1 & 0.3 & t & 0.4 & 1 & 2.0 & 1 & i & 1 & 1.2 & MS, LRI \\
\hline Terpinolene & 1089 & 1 & 0.4 & 1 & 1 & 1 & 1 & 1 & 0.6 & 1 & 1 & 1 & 0.5 & MS, LRI \\
\hline Oxygenated-monoterpenes & & 0.6 & 20.4 & 0.4 & 7.8 & 2.8 & 32.2 & 1.4 & 17.2 & 0.4 & 33.4 & 1.2 & 21.8 & \\
\hline 1.8 Cineole & 1032 & 0.1 & 10.6 & 0.1 & 3.2 & $t$ & 11.2 & 7 & 4.3 & 0.3 & 14.0 & 0.2 & 2.5 & GC,MS.LRI \\
\hline cix-Sabinene hydrate & 1067 & $i$ & 0.1 & $i$ & 0.5 & i & 0.7 & 1 & 0.6 & 0.1 & 1 & 1 & $i$ & MS, LRI \\
\hline Linalool & 1095 & 0.1 & 2.6 & 1 & 1.3 & 2.2 & 10.1 & 0.3 & 4.8 & 1 & 1 & $i$ & 5.5 & GC, MS.LRI \\
\hline trans-Sabinene hydrate & 1098 & 1 & 0.1 & 1 & 0.2 & 1 & 1 & 1 & 1 & 1 & 1 & i & 0.8 & MS, LRI \\
\hline a-Campholenal & 1125 & 1 & 1 & 1 & 0.1 & 1 & 1 & 0.1 & i & $i$ & 0.6 & $i$ & 1 & $\mathrm{MS}, \mathrm{LRI}$ \\
\hline cis-p-Menth-2-en-1-ol & 1139 & 1 & 0.1 & 1 & 1 & 1 & 1 & 1 & 1 & 1 & 1.7 & I & 1 & MS, LRI \\
\hline trans-Pinocarveol & 1143 & 1 & 1 & 1 & 0.2 & 1 & 1.9 & 1 & 1 & 1 & 0.7 & 1 & 1.7 & MS, LRI \\
\hline Pinocarvone & 1155 & 1 & 0.1 & I & 0.1 & j & 0.8 & 0.2 & 0.3 & i & 0.9 & $y$ & 0.8 & GC, MS, LRI \\
\hline cis-Pinocarveol & 1167 & 1 & 1 & 1 & 0.1 & 1 & 1 & 1 & f & 1 & 1 & 1 & 1 & MS, LRI \\
\hline Terpinen-4-ol & 1173 & 1 & 4.2 & 1 & 0.6 & 1 & 0.8 & 0.1 & 1 & 1 & 4.6 & 0.2 & 3.1 & GC, MS, LRI \\
\hline a-Terpineol & 1177 & 0.3 & 0.1 & 0.3 & 0.3 & i & 0.8 & 0.4 & 3.5 & i & 4.4 & 0.2 & 0.7 & GC, MS, LRI \\
\hline Myttenol & 1189 & 1 & 2.0 & 1 & 0.6 & 1 & 2.7 & 1 & 2.0 & 1 & 1.7 & 0.4 & 3.7 & MS, LRI \\
\hline Verbenone & 1219 & 1 & 1 & 1 & 1 & 1 & 1 & 1 & 0.3 & 1 & 1 & 1 & 1 & MS. LRI \\
\hline Cuminaldehyde & 1231 & $i$ & 1 & 1 & 0.1 & 1 & 1 & 1 & 0.3 & 1 & 0.5 & 1 & 1 & MS, LRI \\
\hline cis-Sabinyl acetate & 1262 & 1 & 1 & 1 & 1 & 0.6 & 1 & 1 & 1 & t & 1 & 1 & 1 & MS, L.RI \\
\hline a-Terpinen-7-al & 1265 & 1 & 1 & í & 0.1 & 1 & 0.2 & 1 & 1 & 1 & 1 & 1 & 1 & MS, LRI \\
\hline trans-Pinocarvyl acetate & 1286 & 1 & 1 & 1 & 0.1 & i & 0.3 & 1 & 1 & 1 & 0.3 & $i$ & 1 & MS, LRI \\
\hline cis-pinocarvyl acetate & 1290 & 1 & 1 & 1 & 1 & 1 & 0.1 & 1 & $i$ & , & 0.5 & 1 & 1 & MS, LRI \\
\hline Myrtenyl acetate & 1319 & 1 & 0.5 & 1 & 0.3 & 1 & 2.6 & 0.3 & 1.1 & 1 & 1.5 & 0.2 & 3.0 & GC. MS, LRI \\
\hline 4-Terpinyl acetate & 1325 & 1 & 1 & i & 1 & I & 1 & 1 & 1 & I & 1.3 & $i$ & 1 & GC, MS, LRI \\
\hline a-Tepinyl acetate & 1338 & 1 & 1 & 1 & 1 & 1 & 1 & 1 & 1 & 1 & 0.4 & 1 & 1 & GC, MS, LRI \\
\hline Dihydrocarveol acetate (neoiso) & 1359 & 0.1 & 1 & 1 & 1 & 1 & 1 & 1 & 1 & 1 & 0.3 & 1 & 1 & $M S, L R I$ \\
\hline Sesquiterpenes & & 92.9 & 8.5 & 92.8 & 17.7 & 92.3 & 15.0 & 74.6 & 21.0 & 97.5 & 14.0 & 61.8 & 10.3 & 1 \\
\hline Sesquiterpene hydrocarbons & & 0.7 & 5.5 & 87.5 & 9.4 & 0.9 & 0.4 & 32.8 & 12.3 & 1.6 & 1.0 & 52.0 & 5.2 & 1 \\
\hline a-Copaene & 1371 & 7 & 7 & $t$ & $t$ & 7 & 3 & 0.1 & 7 & 7 & 0.4 & $T$ & 7 & MS, LRI \\
\hline B-Cubebene & 1387 & 1 & 1 & $!$ & $i$ & 0.5 & $i$ & $i$ & $i$ & $y$ & $j$ & 1 & i & MS, LRI \\
\hline B-Elemene & 1400 & 1 & 1 & 0.2 & 0.1 & 1 & 1 & 1 & 1 & 1 & 0.2 & 0.1 & 1 & MS, L.RI \\
\hline Cyperene & 1422 & 1 & 1 & 1 & 0.9 & i & ; & 0.5 & 4.9 & 1 & 1 & 0.4 & 4.4 & MS, LRI \\
\hline a-Gurjunene & 1424 & & & 0.4 & & & & & & & & 0.4 & & MS, LRI \\
\hline (E)-B-Caryophyllene & 1439 & $f$ & 4.8 & 81.4 & 6.4 & 0.2 & 0.4 & 18.4 & 2.9 & 1 & 1 & 47.7 & 0.8 & GC,MS. LRI \\
\hline (Z)-B-famesene & 1446 & 0.3 & i & i & 1 & 1 & 3 & 0.1 & & 0.7 & 1 & 1 & 1 & MS. LRI \\
\hline a-Humulene & 1471 & 1 & 0.3 & 4.3 & 0.4 & i & 1 & 12.4 & 2.1 & it & 0.4 & 3.0 & 1 & GC. MS. LRI \\
\hline$\gamma$-Murrolene & 1478 & 1 & $i$ & 1 & $i$ & 1 & 1 & 0.2 & 1 & 1 & 1 & 1 & 1 & MS. LRI \\
\hline Germacrene-D & 1480 & 1 & ' & 1 & 1 & 1 & 1 & 1 & 1 & 1 & 1 & 1 & 1 & MS, LRI \\
\hline ar-Curcumene & 1481 & 0.1 & 1 & 1 & $i$ & $i$ & 1 & $i$ & $i$ & 0.3 & $i$ & 1 & $i$ & MS. LRI \\
\hline Selina-4,11-diene & 1489 & 1 & 0.1 & 0.3 & 0.4 & 1 & 1 & 0.1 & 0.9 & 1 & 1 & 0.3 & 1 & MS, LRI \\
\hline Eremophyllene & 1513 & 1 & 0.1 & 0.2 & 0.3 & 1 & 1 & 0.5 & 0.4 & 1 & 1 & $!$ & 1 & MS, LRI \\
\hline A-Selinene & 1503 & i & 0.2 & 0.2 & 0.5 & 1 & i & 0.3 & 0.7 & 1 & 1 & 1 & 1 & MS, LRI \\
\hline$(E, E)-\alpha-$ Famesene & 1506 & 0.2 & 1 & 0.1 & 1 & 1 & 1 & 1 & 1 & 0.3 & 1 & 1 & 1 & MS, LRI \\
\hline$\beta$ Bisabolene & 1506 & 0.1 & $i$ & 0.1 & $t$ & $y$ & i & 1 & $i$ & 0.3 & 1 & 1 & 1 & MS, LRI \\
\hline Viridiflorene & 1513 & $i$ & 1 & 0.3 & 0.4 & 1 & 1 & $i$ & 0.4 & 1 & i & $i$ & $i$ & MS, LRI \\
\hline$\partial \overrightarrow{0}$-Cadinene & 1533 & 1 & 1 & 1 & 1 & 0.2 & 1 & 1 & 1 & 1 & I & 0.1 & 1 & MS, LRI \\
\hline a. Cadinene & 1542 & 1 & 1 & 1 & 1 & 1 & 1 & 0.2 & 1 & 1 & 1 & 1 & 1 & MS, LRI \\
\hline Oxygenated-Sesquiterpenes & & 92.2 & 30 & 5.3 & 8.3 & 91.4 & 14.6 & 41.8 & 8.7 & 95.9 & 13.0 & 9.8 & 5.1 & 1 \\
\hline (E)-Nerolidol & 1563 & 91.2 & 7 & 0.3 & 0.3 & 88.0 & 0.3 & 1.3 & 1 & 95.1 & 0.3 & 0.4 & 7 & GC,MS, LRI \\
\hline Caryophyllene oxide & 1600 & 1 & 13 & 3.3 & 4.5 & 1.1 & 8.9 & 23.7 & 2.9 & 1 & 9.9 & 6.2 & 1.0 & GC.MS, LRI \\
\hline Humulene epoxide If & 1623 & $i$ & 0.1 & 0.3 & 0.3 & 1.3 & 0.4 & 10.0 & 1.7 & $i$ & 0.3 & 0.2 & 1.3 & $M S_{\text {, LRI }}$ \\
\hline Caryophylla-4(12),8(13)-dien-5-ol & 1643 & 1 & 1 & 0.1 & 0.1 & 1 & 1.5 & 0.5 & 1 & 1 & 0.3 & 0.3 & 1.1 & MS, LRI \\
\hline a-Bisabolol oxide & 1658 & 0.4 & 1 & 1 & 1 & 1.0 & 1 & 0.4 & 1 & j & 0.8 & 1 & 1 & MS. LRI \\
\hline${ }^{a}$-Selin-11-en-4-ol & 1671 & 1 & 1.6 & 1.1 & 3.0 & 1 & 1.4 & 2.9 & 3.1 & 1 & 0.2 & 2.1 & 1.1 & MS, LRI \\
\hline a-Selin-3,11-dien-6-ol & 1685 & 1 & 1 & 0.1 & 1 & i & 2.1 & 1 & 1 & 1 & 1 & 1 & 1 & MS. LRI \\
\hline a-Bisabolol & 1691 & 0.3 & i & 0.1 & 1 & I & 1 & 1.4 & 0.6 & 0.5 & 1.2 & 0.6 & $i$ & GC,MS. LRI \\
\hline Cyperenone & 1709 & $j$ & 1 & $i$ & 0.1 & 1 & 1 & 0.7 & 0.4 & 1 & 1 & $j$ & 0.6 & MS, LRI \\
\hline (2E, GE)-Famesol* & 1722 & 0.2 & 1 & 1 & 1 & t & 1 & 0.2 & 1 & 0.3 & 1 & 1 & 1 & MS, LRI \\
\hline$(2 E, 6 E)$-Farnesyl acetate & 1822 & 0.1 & 1 & 1 & 1 & 1 & 1 & 0.7 & 1 & 1 & 0.9 & 1 & $i$ & MS, LRI \\
\hline Aromatic compounds & & 1.0 & 0 & 0 & 0 & 0.3 & 0 & 0 & 0.0 & 0.0 & 0.4 & 0 & 0 & 1 \\
\hline o-guaiacol & 1089 & 0.2 & $T$ & 1 & 7 & 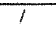 & 7 & $T$ & 1 & $T$ & 1 & $T$ & $T$ & MS, LRI \\
\hline 2-Phenylethyl acctate & 1259 & 0.5 & I & i & $i$ & 0.3 & 1 & 1 & 1 & 1 & 0.4 & 1 & 1 & MS, LRI \\
\hline (D)-cinnamyl acetate & 1452 & 0.3 &  & 1 & 1 & 1 & 1 & 1 & 1 & 1 & 1 & 1 & 1 & GC, MS, LRI \\
\hline Aliphatic compounds & & 3.7 & 0 & 0 & 0 & 1.5 & 0 & 0 & 0 & 0.2 & 0 & $\theta$ & 0 & 1 \\
\hline 2-heptanol & 869 & 0.1 & T & 7 & 7 & 7 & $t$ & 7 & 7 & 1 & 7 & $i$ & 1 & MS, LRI \\
\hline 2-Heptyl acetate & 1042 & 2.8 & 1 & 1 & 1 & 0.7 & 1 & $t$ & $i$ & 1 & $i$ & 1 & 1 & MS, LRI \\
\hline Octyl acetate & 1196 & 0.1 & 1 & 1 & 1 & 0.8 & 1 & 1 & 1 & 0.2 & 1 & 1 & 1 & MS, LRI \\
\hline Decyl acetate & 1408 & 0.7 & 1 & 1 & 1 & 1 & 1 & 1 & 1 & 1 & 1 & 1 & 1 & MS. LRI \\
\hline Total & & 99.2 & 99.6 & 97.8 & 97.4 & 96.9 & 97.9 & 91.9 & 96.2 & 99.7 & 97.3 & 96.5 & 89.7 & 1 \\
\hline
\end{tabular}

i: traces (<0,1). Se: Seeds; Pe: Pericarps; Le; Leaves; Rh: Rhizomes; ${ }^{*}$ correct isomer not identified; LRI: Linear retention Index; Identification methods: GC, identification based on co-injection with authentic sample; MS. identification based on comparison of mass spectrum with literature data; LRI, identification based on comparison of retention index with those of published data. 
Table 3: Correlation between chemical composition (relative percentages of the major constituents) and antibacterial activity (MIC and $\mathrm{MBC}$ in $\mu \mathrm{L} / \mathrm{mL}$ ) of the essential oils of $A$ dalielit, A. letestitomum and A. pruinosum and of their major components

\begin{tabular}{|c|c|c|c|c|c|c|c|c|c|c|c|c|c|c|c|c|}
\hline & \multicolumn{4}{|c|}{ Antibacterial activity of pure compounds } & & \multirow{2}{*}{\multicolumn{11}{|c|}{ Chemical composition (\%) }} \\
\hline & \multicolumn{2}{|c|}{ M. luteus } & \multicolumn{2}{|c|}{ E. coli } & \multirow{2}{*}{\multicolumn{4}{|c|}{ A. datzielii }} & & & & & & & & \\
\hline & & & & & & & & & \multicolumn{4}{|c|}{ A. letestuianum } & \multicolumn{4}{|c|}{ A. pruinosum } \\
\hline & $\mathrm{MIC}$ & $\mathrm{MBC}$ & $\mathrm{MIC}$ & MBC & Se & $\mathrm{Pe}$ & Le & $\mathbf{R h}$ & Se & $\mathrm{Pe}$ & Le & $\mathrm{Rh}$ & $\mathrm{Se}$ & $\mathrm{Pe}$ & Le & $\mathrm{Rh}$ \\
\hline Sabinene & $50 \mathrm{c}$ & $>50$ & $1.56 \mathrm{~b}$ & 6.25 & - & 14.2 & $t$ & 22.9 & $=$ & 0.8 & -3 & 11.6 & $\cdot$ & 2.9 & 0.3 & 9.8 \\
\hline B-Pinene & $3.12 b$ & 12.5 & $0.19 \mathrm{a}$ & 0.78 & - & 42.3 & 3.9 & 37,9 & - & 38.5 & 13.4 & 32.9 & - & 29.0 & 27.8 & $\mathbf{3 4 . 3}$ \\
\hline 1.8-Cineole & $12.5 \mathrm{c}$ & $>50$ & $0.78 \mathrm{a}$ & 3.12 & 0.1 & 10.6 & 0.1 & 3.2 & $t$ & 11.2 & 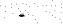 & 4.3 & 0.3 & 14,0 & 0.2 & 2.5 \\
\hline Linalow & $0.1 \% \mathrm{a}$ & 0.78 & $0.19 \mathrm{a}$ & 0.19 & 0.1 & 2.6 & - & 1.3 & 2.2 & 10.1 & 0.3 & 4.8 & $\cdot$ & - & -1 & 5.5 \\
\hline (E)-B-Caryophyllene & $>50 \mathrm{c}$ & $>50$ & $50 \mathrm{c}$ & $>50$ & - & 4,8 & & 6.4 & 0.2 & 0.4 & 18.4 & 29 & - & - & 47.7 & 0.8 \\
\hline a-tumulene & $>50 c$ & 250 & $6.25 b$ & $>50$ & - & 0.3 & 4.3 & 0.4 & - & $\because$ & 12.4 & 21 & - & 0.4 & 3.0 & $\because$ \\
\hline (b)-Nerolidol (synhetic) & $0.19 \mathrm{a}$ & 0.19 & $0.19 \mathrm{a}$ & 0.19 & - & - & - & - & - & . & - & . & - & - & - &. \\
\hline (R) $(E)$ Nerolidol (natural) & $0.19 \mathrm{a}$ & 0.19 & $0.19 \mathrm{a}$ & 0.19 & 91.2 & - & 0.3 & 0.3 & 88.0 & 0.3 & 1.3 & $\approx$ & 95.1 & 0.3 & 0.4 & - \\
\hline Caryophyllene oxide & $12.5 \mathrm{e}$ & $>50$ & $25 c$ & $>50$ & $\therefore$ & 1.3 & 3.3 & 4.5 & 1.1 & 8.9 & 23.7 & 2.9 & - & 9.0 & 6.2 & 1.0 \\
\hline \multirow{4}{*}{$\begin{array}{l}\text { Antibacterial } \\
\text { activity of } \\
\text { essentials oils }\end{array}$} & \multirow{2}{*}{\multicolumn{2}{|c|}{ M. Iuteus }} & \multicolumn{2}{|c|}{ MIC } & $0.39 \mathrm{a}$ & $25 \mathrm{c}$ & $25 \mathrm{c}$ & $12.5 \mathrm{c}$ & $0.39 \mathrm{a}$ & $25 \mathrm{c}$ & $078 \mathrm{a}$ & $12.5 \mathrm{c}$ & $0.78 \mathrm{a}$ & $12.5 \mathrm{c}$ & $25 \mathrm{e}$ & $50 \mathrm{c}$ \\
\hline & & & \multicolumn{2}{|c|}{ MBC } & 25 & $>50$ & 50 & 50 & 0.78 & 50 & 6.25 & 25 & 12.5 & 12.5 & 25 & 50 \\
\hline & \multirow{2}{*}{\multicolumn{2}{|c|}{ E. coli }} & \multirow{2}{*}{\multicolumn{2}{|c|}{$\begin{array}{l}\text { MIC } \\
\text { MBC }\end{array}$}} & $0.19 \mathrm{a}$ & $1.56 \mathrm{~b}$ & $1.56 b$ & $0.19 \mathrm{a}$ & $0.39 a$ & $0.19 a$ & $0.78 \mathrm{a}$ & 1.566 & $0.19 a$ & $0.78 \mathrm{a}$ & $1.56 \mathrm{~b}$ & $0.19 \mathrm{a}$ \\
\hline & & & & & 1.56 & 6.25 & 12.5 & 3.12 & 0.39 & 1.56 & 3.12 & 625 & 3.12 & 3.12 & 12.5 & 1,56 \\
\hline
\end{tabular}

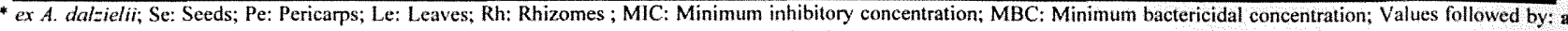
("strong activity"). MIC ranging from $0.19-0.78 \mu \mathrm{L} / \mathrm{mL} ; \mathbf{b}$ ("moderate activity"), MIC ranging from $1.56-6.25 \mu \mathrm{L} / \mathrm{mL} ; \mathbf{c}$ ("low activity"), MIC ranging from $12.5-50 \mu \mathrm{L} / \mathrm{mL}$ :

The antibacterial activities should be expected to relay the chemical compositions of the essential oils, the structural configuration of the components of the oil, their functional groups and possible synergistic interactions between components [18, 19]. Single compounds, identified as major components of the tested essential oils, were then tested under the same experimental conditions against both microorganisms. Components with an alcohol functional group such as $(E)$-nerolidol and linalool were highly active against both microorganisms; the synthetic and purified nerolidol showing the same efficiency, contrary to the observations of Schmidt et al. [20] on Staphylococcus aureus with synthetic cisnerolidol and natural trans-nerolidol. Furthermore, Brehm-Stecher \& Johnson [21] showed that nerolidol induces a sensitization of Staphylococcus aureus and Escherichia coli to antibiotics. A strong bactericidal activity $(\mathrm{MBC}=0.19 \mu \mathrm{L} / \mathrm{mL})$ was observed with linalool and nerolidol for $E$. coli and with nerolidol in the case of $M$. luteus. On the other hand, $(E)$ - $\beta$-caryophyllene and its epoxide showed the lowest activity ( $\mathrm{MIC}=12.5$ to $50 \mu \mathrm{L} / \mathrm{mL}$ ); among the other tested compounds, $\beta$-pinene was the most active against $E$. coli $(\mathrm{MIC}=0.19 \mu \mathrm{L} / \mathrm{mL})$, while sabinene and $\alpha$-humulene were moderately active against the same microorganism (1.56 and 6.25 $\mu \mathrm{L} / \mathrm{mL}) ; 1,8$-cineole exhibited a good antibacterial activity on $E$. coli $(\mathrm{MIC}=0.78 \mu \mathrm{L} / \mathrm{mL})$.

These results are in accordance with previous results that indicated that oxygenated compounds exhibited the strongest antimicrobial activities, especially visible on whole cells, while hydrocarbon derivatives were less active because of their limited hydrogen bound capacity and water solubility [7]. Nevertheless, a high bactericidal activity was reported for terebenthine oil, which was attributed to pinenes [22, 23]. 1,8-Cineole was previously reported for its potential antimicrobial activity, either alone or in combination with other minor compounds in essential oils [24]. The strong activities observed against $E$. coli for the rhizome oils of $A$. dalziellii and $A$. pruinosum, as well as for the pericarps oil of $A$. letestuianum, might be related to their high content of $\beta$-pinene, in combination with sabinene and 1,8-cineole or linalool.

In conclusion, regarding the relationship between the antibacterial activities of Aframomum essential oils and their main components, the strong activities of the seed oils against both microorganisms might be attributed to their high content of $(R)$ - $(E)$-nerolidol; these essential oils could then be used as agro-alimentary products owing to their aromatizing and bactericidal properties and could be included in effective and inexpensive formulations to reduce the presence of pathogens in food in place of conventional chemical antimicrobials. Finally, our study corroborates the use of Aframomum seeds in traditional culinary preparations.

\section{Experimental}

Plant material and extraction procedure: All plant materials (seeds, pericarps, leaves and rhizomes) were collected in the western area of Cameroon; both $A$. dalzielii and A. pruinosum were harvested in August 2008 at N'jenseh and Mbelah village of Fontem, respectively, while $A$. letestuianum samples were collected in March 2008 at Bamendjida, a village of Mbouda. The botanical identifications were carried out at the National Herbarium of Cameroon where voucher specimens are kept: NKM $165639 / \mathrm{HNC}$, $45194 \mathrm{HNC}$ and $43138 \mathrm{HNC}$, respectively. A portion $(200 \mathrm{~g})$ of each plant material was submitted for $6 \mathrm{~h}$ to hydrodistillation, using a Clevenger-type apparatus. The obtained essential oils, separated from water by decantation, were dried over anhydrous sodium sulfate and, after filtration, stored in sealed flasks at $4^{\circ} \mathrm{C}$ until tested and analyzed. The yields $(w / w)$ were calculated according to the weight of fresh plant material (Table 1).

Chemical analysis: The essential oils were analyzed by gas chromatography (GC) and gas chromatography combined with mass spectrometry (GC-MS).

Gas chromatography analysis: GC analyses were performed on a Varian gas chromatograph, model CP-3380, equipped with a flame ionization detector (FID) using an HP-5 J\&W Agilent (5\% phenyl$95 \%$ methylpolysiloxane) capillary column (30 $\mathrm{m} \times 0.25 \mathrm{~mm}$, film thickness $0.25 \mu \mathrm{m}$ ). Injector and detector temperatures were set at 220 and $250^{\circ} \mathrm{C}$, respectively. Oven temperature was gradually raised from $50^{\circ} \mathrm{C}$ to $200^{\circ} \mathrm{C}$ at $5^{\circ} \mathrm{C} / \mathrm{min}$, then kept at $200^{\circ} \mathrm{C}$ for 10 min. $\mathrm{N}_{2}$ was the carrier gas, at a flow rate of $0.8 \mathrm{~mL} / \mathrm{min} ; 0.1 \mu \mathrm{L}$ of pure sample was injected manually and in a split mode (1:100).

GC-chiral analyses of racemic $(E)$-nerolidol, cabreuva oil containing mainly $(+)-(E)$-nerolidol (kindly afforded by "Laboratoire Rosier Davenne", Avignon, France) and $(-)-(E)$ nerolidol isolated from A. dalzielii, were carried out on a HP 5890 provided with a Cyclosil-B (J\&W ref $112-6632$ ) capillary column (30\% 6-TBDMS-2,3-dimethyl $\beta$-cyclodextrine) having the following dimensions: $30 \mathrm{~m} \times 0.25 \mathrm{~mm}$ i.d. $\times 0.25 \mu \mathrm{m}$ film. The temperature program was: $80^{\circ} \mathrm{C}$, held for $5 \mathrm{~min}$, raising at $1^{\circ} \mathrm{C} / \mathrm{min}$ to $130^{\circ} \mathrm{C}$, then at $4^{\circ} \mathrm{C} / \mathrm{min}$ to $175^{\circ} \mathrm{C}$, finally kept at $175^{\circ} \mathrm{C}$ during 4 min; injection volume: $0.2 \mu \mathrm{L}$ of a $10: 100 \mathrm{CH}_{2} \mathrm{Cl}_{2}$ solution (split ratio 1:100); carrier gas $\mathrm{He}$ at a constant pressure $10 \mathrm{psi}$, injector temperature $250^{\circ} \mathrm{C}$, flame ionization detector temperature $250^{\circ} \mathrm{C}$. 
Gas chromatography-mass spectrometry: GC-MS analyses were performed using a Hewlett Packard 5890 II gas chromatograph, interfaced with a quadrupole detector (Model 5972) and equipped with a HP-5 MS capillary column $(30 \mathrm{~m} \times 0.25 \mathrm{~mm}$, film thickness $0.25 \mu \mathrm{m}$ ). Helium was the carrier gas, at a flow rate of $0.6 \mathrm{~mL} / \mathrm{min}$. Injector and MS transfer line temperatures were $220^{\circ} \mathrm{C}$ and $250^{\circ} \mathrm{C}$, respectively. The oven programme temperature was the same as that used in the $\mathrm{GC}$-FID analyses. Diluted samples (10:100 in $\mathrm{CH}_{2} \mathrm{Cl}_{2}$, $v / v)$ of $1 \mu \mathrm{L}$ were injected manually and in a split mode (1:100). The MS was operated in the EI mode at $70 \mathrm{eV}$, in the $\mathrm{m} / \mathrm{z}$ range $35-$ 300 ; electronmultiplier $1460 \mathrm{eV}$; scan rate, $2.96 \mathrm{scan} / \mathrm{s}$.

Qualitative analysis: The identification of the constituents was assigned on the basis of a comparison of their relative retention indices, calculated with reference to a series of $n$-alkanes $\left(\mathrm{C}_{9}-\mathrm{C}_{20}\right)$, and their mass spectra with those of the standards (for main components), those found in the literature [25] and supplemented by the NBS75K database and Wiley $7^{\text {th }}$ NIST 98 EPA/NIH Mass Spectral Library Upgrade (provided by Hewlett Packard with the GC/MS control and data processing software).

Quantification: The relative proportions of the essential oil constituents were obtained electronically from the GC-FID data by peak area normalization, taking response factors (RRF) as 1.0 for all compounds. Accurate quantification of the main component of the seed essential oils, $(E)$-nerolidol, was carried by the internal standard method. The response factor RRF of $(E)$-nerolidol (target) was calculated by using the following equation and adopting tridecane as internal standard (i.s. $): R R F=\left(C_{\text {target }} \times A_{i . s .}\right) /\left(A_{\text {target }} \times\right.$ $\left.C_{i . s}\right)$, where $C_{\text {target }}$ is the concentration of the referent solution, $A_{\text {target }}$ its absolute peak area, $C_{i . s .}$ the concentration of the internal standard and $A_{i . s .}$ its absolute peak area [26]. A graphical representation expressing the area ratios $A_{i . s} / A_{\text {target }}$ depending on the concentration ratio $C_{\text {i.s. }} / C_{\text {target }},\left(C_{i . s}=2.5 \mathrm{~g} / 1, C_{\text {target }}\right.$ ranging from 1 to $10 \mathrm{~g} / \mathrm{L}$ in $n$ hexane) could be achieved; the calibration curve was linear over the concentrations tested, according to the regression equation $y=1.1558 x-0.0099$ in which the slope corresponds to the response factor. The mean RRF value ( 3 replicates) calculated under our experimental conditions was: $R R F=1.16+/-0.06$. Finally, contents of $(E)$-nerolidol in the 3 seed essential oils, expressed as $\mathrm{g} / 100 \mathrm{~g}$ of essential oil, were calculated as: weight $\%=A_{\text {target }} \times m_{\text {i.s. }} \times R R F \times$ $100 / A_{i, s} \times m_{\text {oil }}$ in which $m_{i, s}$ and $m_{\text {oil }}$ are expressed as $\mathrm{g}$.

Isolation of $(E)-(R)-n e r o l i d o l$ by liquid solid chromatography and characterization: Seed oil of $A$, dalzielii $(1 \mathrm{~g})$ was loaded onto a glass column packed with $60 \mathrm{~g}$ of silica gel $60 \mathrm{~A}(35-70 \mu \mathrm{m})$ as a slurry in $n$-hexane. Elution was performed with increasing polarity of the solvent mixture ( $n$-hexane: diethyl ether 95:5; 90:10;80:20; 70:30); solvents were dried and distilled before use. All fractions were screened by GC-FID and GC-MS analyses; those containing the single major constituent were gathered then concentrated to yield $0.82 \mathrm{~g}$ of pure $(-)-(E)$-nerolidol, a colorless liquid with a pleasant floral odor.

Optical rotation: The specific optical rotation was measured in $\mathrm{CHCl}_{3}(c 0.1)$ at $25^{\circ} \mathrm{C}$ using a Perkin Elmer 241 polarimeter.
NMR data: ${ }^{1} \mathrm{H}$ and ${ }^{13} \mathrm{C}$ NMR spectra of nerolidol were recorded with a Bruker DRX-400 spectrometer using $\mathrm{CDCl}_{3}$ as solvent and TMS as internal standard (400 and $100 \mathrm{MHz}$, respectively). The assignment of ${ }^{1} \mathrm{H}$ and ${ }^{13} \mathrm{C}$ signals was supported by one- and twodimensional NMR experiments. 2D-NMR spectra were obtained from standard Brüker software.

Antimicrobial activities: Commercial chemical compounds : sabinene (Fluka, 84085, purity of 99\%), $\beta$-pinene (Aldrich, 80609, purity of $98 \%$ ), 1,8-cineole (Fluka, 29210, purity of 98\%), linalool (Fluka, 74856, purity of 97\%), (E)- $\beta$-caryophyllene (Fluka, 22075, purity of $98.5 \%$ ), $\alpha$-humulene (Extrasynthèse, $5424 \mathrm{~S}$, purity $>95 \%$ ), synthetic (E)-nerolidol (Fluka 18143, purity $>85 \%$ ), caryophyllene oxide (Interchim, $0 \mathrm{C} 125765$, purity of $95 \%$ ).

The antibacterial activity of the essential oils and pure molecules were tested against Micrococcus luteus ATCC 4698 (Grampositive) and Escherichia coli 363 ATCC 11775363 (Gramnegative) using a broth dilution method [27]. Minimum inhibitory concentration (MIC) and minimum bactericidal concentration (MBC) were determined for all samples. Stock solutions of essential oils, of 8 authentic commercial compounds (sabinene, $\beta$-pinene, 1,8-cineole, linalool, synthetic $(E)$-nerolidol (racemic mixture), $(E)$ $\beta$-caryophyllene, $\alpha$-humulene, caryophyllene oxide), as well as of the isolated $(E)-(R)$-nerolidol, were prepared by geometric dilutions in sterile distilled water starting from a $1 / 2$ to $1 / 512$ dilutions, with vigorous shaking between each dilution. Then $100 \mu \mathrm{L}$ of each solution was added to $900 \mu \mathrm{L}$ of the bacterial culture previously prepared (the optical density of the inoculum was measured at $D_{600}$ $=0.1$ and then diluted to reach a final optical density of $D_{600}=0.001$ in the assay), to reach a final concentration of the tested ingredient ranging from 0.19 to $50 \mu \mathrm{L} / \mathrm{mL}$. The positive control was obtained with the bacterial culture without the antimicrobial agent, the negative control containing only the medium. After over-night incubation at $30^{\circ} \mathrm{C}$ with constant shaking $(300 \mathrm{rpm}), 100 \mu \mathrm{L}$ of each dilution was transferred to microplates, then optical density was measured at $600 \mathrm{~nm}$. The MIC, defined as the lowest concentration of the essential oil or pure compound at which the microorganism did not demonstrate visible growth, was evaluated by testing serial triplicate dilutions.

Cell suspensions $(0.1 \mathrm{~mL})$ from the tubes showing no growth were subcultured on Zobell and Poor broth agar plates to determine if the inhibition was reversible or permanent. $\mathrm{MBC}$ was determined as the highest dilution (lowest concentration) at which no growth occurred on the agar plates.

Acknowledgements - Authors are grateful to the "French Cooperation-Cameroon", the Francophonie University Agency East Africa Office (AUF) for the financial support. We are also thankful to Mr Senou Guy and Joseph Kwanga for the facilities provided for collecting samples in Fontem and Bamendjida, as well as to Mr Mesili Paul for the authentication of Aframomum dalzielii (National Herbarium of Cameroon).

\section{References}

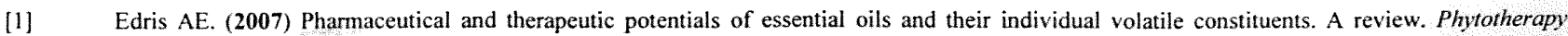
Research, 21, 308-332.

[2] Adorjan B, Buchbauer G. (2010) Biological properties of essential oils: an updated review. Flavour and Fragrance Journal, 25, 407-426.


Immunologiae Therapiae Experimentalis, 55, 315-327.

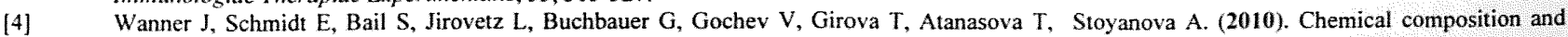
antibacterial activity of selected essential oils and some of their main compounds. Natural Product Communications, 5 , $1359-1364$. 
Lang G, Buchbauer G. (2012) A review on recent research results (2008-2010) on essential oils as antimicrobials and antifungals. Flavour and Fragrance Journal, 27, 13-39.

Burt S. (2004) Essential oils: Their antibacterial properties and potential applications in foods. A review. International Journal of Food Microbiology, 94, 223-253.

Sokovic M, Marin PD, Brkic D, Van Griensven LJLD. (2008) Chemical composition and antibacterial activity of essential oils of ten aromatic plants against human pathogenic bacteria. Food, 1, 220-226.

Shelef LA. (1983) Antimicrobial effects of spices. Journal of Food Safety, 6, 29-44.

(a) Amvam Zollo PH, Abondo R, Agnaniet H, Fekam F, Bessiere JM, Menut C. (2005) Traditional uses of Aframomum species growing in Cameroon and chemical analysis of their volatiles. In Processing, analysis and application of essential oils. Jirovetz L, Buchbauer G. (Eds). Har Krishan Bhalla \& Sons, India, 169-207; (b) Amvam Zollo PH, Abondo R, Biyiti L, Menut C, Bessiere JM. (2002) Aromatic plants of tropical Central Africa. XXXVIII. Chemical composition of the essential oils from four Aframomum species collected in Cameroon. Journal of Essential Oil Research, 14, 95-98.

Agnaniet H, Menut C, Bessiere JM. (2004) Aromatic plants of Tropical and Central Africa. Part XLIX: Chemical composition of essential oils of the leaf and thizome of Aframomum giganteum K. Schum. from Gabon. Flavour and Fragrance Journal, 19, 205-209.

(a) Menut C, Lamaty G, Amvam Zollo PH. (1991) Aromatic plants of Tropical and Central Africa V: Volatile components of three Zingiberaceae from Cameroon: Aframomum melegueta (Roscoe) K. Schum., A. daniellii (Hook. F.) K. Schum. and A. sulcatum (Oliv. and Hanb.) K. Schum. Flavour and Fragrance Journal, 6, 183-186; (b) Menut C, Lamaty G, Amvam Zollo PH, Abondo R., Bessiere JM. (1994) Aromatic plants of Tropical and Central Africa. XII. Fruit essential oil of Aframomum pruinosum Gagnepain. A potential source of $(E)-(R)$-Nerolidol. Journal of Essential Oil Research, 6, 13-16.

Smith RM. (1982) Analysis of the pungent principles of ginger and grains of paradise by high-performance liquid chromatography using electrochemical detection. Chromatographia, 16, 155-157.

Tane P, Tatsimo SD, Ayimele GA, Connolly JD. (2005) Bioactive metabolites from Aframomum species. $11^{\text {th }}$ NAPRECA Symposium Book of Proceedings, Antananarivo, Madagascar, 214-223.

Surburg H, Panten J. (2006) Common fragrance and flavors materials. Preparation, properties and uses, $5^{\text {th }}$ completely revised and enlarged edition, Wiley-VCH Verlag GmbH \& Co., KGaA, Weinheim.

Schubert V, Dietrich A, Ulrich T, Mosandl A. (1992) The stereoisomers of nerolidol: Separation, analysis and olfactoric properties. Zeitschrift fïr Naturforschung, 47c, 304-307.

Yang K, Zhou YX, Wang CF, Du SS, Deng ZW, Liu QZ, Liu ZL. (2011) Toxicity of Rhododendron anthopogonoides essential oil and its constituent compounds towards Sitophilus zeamais. Molecules, 16, 7320-7330.

Kaiser R. (2006) Meaningful scents around the World: Olfactory, chemical, biological, and cultural considerations. Verlag Helvetica Chimica ActaZürich, ed. WILEY-VCH, 17-19.

Trombetta D, Castelli F, Sarpietro MG, Venuti V, Cristani M, Daniele C, Saija A, Mazzanti G, Bisignano G. (2005) Mechanisms of antibacterial action of three monoterpenes. Antimicrobial Agents and Chemotherapy, 49, 2474-2478.

Helander IM, Alakomi H-L, Kyösti L-K, Mattila-Sandholm T, Pol I, Smid EJ, Gorris JGM, von Wright A. (1998) Characterization of the action of selected essential oils components on Gram-negative bacteria. Journal of Agricultural Food Chemistry, 46, 3590-3595.

Schmidt E, Bail S, Friedl SM, Jirovetz L, Buchbauer G, Wanner J, Denkova Z, Slavchev A, Stoyanova A, Geissier M. (2010) Antimicrobial activities of single aroma compounds. Natural Product Communications, 5, 1365-1368.

Brehm-Stecher BF, Jonhson EA. (2003) Sensitization of Staphylococcus aureus and Escherichia coli to antibiotics by the sesquiterpenoids nerolidol, famesol, bisabolol and apritone. Antimicrobial Agents and Chemotherapy, 47, 3357-3360.

Mercier B, Prost J, Prost M. (2009) L'huile essentielle de térébenthine et sa partie la plus volatile ( $\alpha$ - et $\beta$-pinenes): Une revue bibliographique. International Journal of Occupational Medicine and Environmental Health, 22, 331-342.

Sikkema J, de Bont JA, Poolman B. (1994) Mechanisms of membrane toxicity of hydrocarbons. Microbiological Reviews, 59, $201-222$.

Randrianarivelo R., Sarter S, Odoux E, Brat P, Lebrun M, Romestand B, Menut C, Andrianoelisao HS, Raherimandimby M, Danthu P. (2009) Composition and antimicrobial activity of essential oils of Cinnamosma fragrans. Food Chemistry, 114, 680-684.

Adams RP. (2007) Identification of Essential Oil Components by Gas. Chromatography/ Mass Spectrometry. $4^{\text {th }}$ Edition. Allured Publishing Corporation, Carol Stream, Il 60188-2787, USA.

Cicchetti E, Merle P, Chaintreau A. (2008) Quantitation in gas chromatography: usual practices and performances of a response factor database. Flavour and Fragrance Journal, 23, 450-459.

Hancock REW. (2001) Cationic peptides: effectors in innate immunity and novel antimicrobials. The Lancet Infections Diseases, I, 156-164. 